Digital Press Social Sciences and Humanities

Intuition linguistique et stratégie de l'apprentissage des étudiants « faibles »

Dwi Astuti, Sudarwoto and Durrotum Millatina

Proceeding of Conférence internationale sur le français 2018

Joesana Tjahjani, Merry Andriani, Sajarwa, Wening Udasmoro (eds) 


\title{
Intuition linguistique et stratégie de l'apprentissage des étudiants « faibles »
}

\author{
Dwi Astuti*, Sudarwoto et Durrotum Millatina \\ Universitas Negeri Semarang, Semarang, Indonesia \\ *e-mail : astutidwi@mail.unnes.ac.id
}

\section{Résumé}

La réalité depuis des années montre qu'au département de pédagogie de français, il y a toujours des étudiants "faibles". Bien qu'ils soient finalement diplômés, ils doivent répéter des sujets de compétences linguistiques à plusieurs reprises et passer leurs études jusqu'à 14 semestres. Alors, il faut trouver un moyen de les aider pour améliorer leurs compétences et à raccourcir leurs périodes d'études. Une des façons qu'on peut faire est de connaître d'abord leurs intuitions linguistiques et leurs stratégies d'apprentissage. Cette étude vise à décrire les intuitions linguistiques et les stratégies d'apprentissage utilisées par les étudiants du département de pédagogie de français qui sont "faibles". Cette recherche est une étude descriptive. Les données sur les tests d'intuitions linguistiques sont obtenues sur la capacité de réflexion verbale et la capacité de réflexion abstraite, et les données sur les stratégies d'apprentissage sont obtenues à partir de questionnaires. Les résultats ont montré qu'au test de capacité de réflexion verbale et au test de la réflexion abstraite, $50 \%$ des répondants étaient inclus dans la catégorie de Basse, tandis que les stratégies d'apprentissage utilisées par les répondants sont la stratégie de la mémoire, cognitive, de la compensation, métacognitive, affective, et sociale. La stratégie la plus utilisée est celle de cognitive et la moins utilisée est celle de sociale.

\section{Mots-clés}

intuition linguistique, stratégie d'apprentissage, étudiant "faible"

\begin{abstract}
The reality for years has shown that in the French pedagogy department, there are always "weak" students. Although they have finally graduated, they must repeat language skills subjects several times and spend their studies up to 14 semesters. So we have to find a way to help them improve their skills and shorten their periods of study. One of the ways to do this is to first learn about their linguistic intuitions and learning strategies. The purpose of this study is to describe the linguistic intuitions and learning strategies used by students in the French pedagogy department who are "weak". This research is a descriptive study. Data on language intuition tests are obtained on verbal thinking skills and abstract thinking skills, and data on learning strategies are obtained from questionnaires. The results showed that $50 \%$ of respondents were included in the low-income category of the verbal reflection and abstract thinking tests, while the learning strategies used by respondents are memory, cognitive, compensation, metacognitive, emotional, and social. The most widely used strategy is cognitive and the least used is social.
\end{abstract}

\section{Keywords}

linguistic intuition, learning strategy, "weak" student 


\section{Introduction}

La réalité depuis des années montre qu'au département de pédagogie de français, il y a toujours des étudiants "faibles". Bien qu'ils soient finalement diplômés, l'indice de réussite qu'ils atteignent, n'est pas obtenu à partir du cours dans l'apprentissage de langue. Elle atteint 2,5 ou plus, car il est soutenu par les notes des cours comme la Religion, la Langue Indonésienne, l'Introduction à la Culture, les Stratégies d'Enseignement et d'Apprentissage. Par exemple, les étudiants du département de pédagogie de français de l'Universitas Negeri Semarang l'année 2016, parmi 22 étudiants guidés par un conférencier, 5 (environ $20 \%$ ) ont les notes aux sujets de compétences linguistiques au premier semestre comme suit :

Tableu 1 Les notes aux sujets de compétences linguistiques au premier semestre

\begin{tabular}{|l|l|l|l|l|l|l|l|l|}
\hline \multirow{2}{*}{ Nom } & Note & \multicolumn{5}{|c|}{} & & $\begin{array}{l}\text { Indice de } \\
\text { réussite }\end{array}$ \\
\cline { 3 - 9 } & & COPE & CEPE & POPE & PEPE & GRAM & PHON & CH \\
\hline SPL & & E & C & E & E & CD & D & 0,75 \\
\hline HAS & & CD & D & E & D & E & B & 1,25 \\
\hline MO & & E & C & D & CD & BC & CD & 1,42 \\
\hline ZW & & CD & CD & E & CD & E & C & 1,08 \\
\hline AAI & & E & E & E & E & E & CD & 0,25 \\
\hline
\end{tabular}

Explication :
COPE $\quad$ : Compréhension Orale Pré Élémentaire
CEPE $\quad:$ Compréhension Écrite Pré Élémentaire
POPE $\quad:$ Production Orale Pré Élémentaire
PEPE $\quad:$ Production Écrite Pré Élémentaire
GRAM $\quad:$ Pratique de la Grammaire Pré Élémentaire
PHON $\quad:$ Correction Phonétique et Prononciation

Ils obtiennent les notes $\mathrm{CD}$, D et $\mathrm{E}$ dans les cours de français, ce sont : la compréhension orale, l'expression orale, la compréhension écrite, la production écrite, la grammaire, la phonétique et la prononciation.

Dans les cours générales ou l'éducation, on peut dire que leurs notes sont bonnes. Les données suivantes illustrent cela.

Tableu 2 Les notes des cours générales ou l'éducation

\begin{tabular}{|c|c|c|c|c|c|}
\hline \multirow[t]{2}{*}{ Nom } & \multicolumn{4}{|c|}{ Note } & \multirow{2}{*}{$\begin{array}{l}\text { Indice de } \\
\text { réussite }\end{array}$} \\
\hline & $\mathrm{PE}$ & LI & A & EC & \\
\hline SPL & B & B & $\mathrm{BC}$ & A & 3,13 \\
\hline HAS & $\mathrm{AB}$ & $B$ & $\mathrm{BC}$ & $\mathrm{AB}$ & 3,13 \\
\hline MO & B & $\mathrm{AB}$ & $\mathrm{B}$ & $\mathrm{AB}$ & 2,75 \\
\hline ZW & $\mathrm{AB}$ & B & $B$ & $\mathrm{AB}$ & 2,75 \\
\hline AAI & $\mathrm{A}$ & $B$ & $\mathrm{C}$ & $\mathrm{A}$ & 3,25 \\
\hline
\end{tabular}

Explication :

$\begin{array}{ll}\text { PE } & : \text { Pancasila Éducation } \\ \text { LI } & : \text { Langue Indonésienne } \\ \text { A } & : \text { Anglais } \\ \text { EC } & : \text { Éducation à la Conservation }\end{array}$

Avec cette condition, et en plus il n'y a pas de système de chute, ces étudiants peuvent obtenir le certificat de fin d'études, bien qu'ils doivent répéter des sujets de compétences linguistiques à plusieurs reprises et passer leurs études jusqu'à 14 semestres. Parfois, au dernier semestre, les enseignants sont "forcés" de les aider pour qu'ils ne soient pas drop-out.

Considérant que les étudiants "faibles" sont toujours présents dans chaque année, ils nécessitent une attention et une manipulation particulières, de sorte que la qualité des diplômés est protégée. Ils ont des obstacles psychologiques qui entraînent un échec scolaire. Les obstacles psychologiques est se ressentir ou reconnaitre qu'ils sont faibles et qu'ils ont perdu confiance. En conséquence, ils ne veulent pas essayer, 
car ils ont peur de ne pas réussir. L'une des façons qu'on peut faire de surmonter ces obstacles est de connaître leur intuition linguistique et les stratégies d'apprentissage qu'elles appliquent.

Basé sur la description ci-dessus, il est nécessaire d'étudier les intuitions linguistiques et les stratégies d'apprentissage des élèves "faibles" au département de pédagogie de français, et plus tard, on peut concevoir un modèle approprié pour les aider. Ce modèle contient une approche psychologique qui vise à améliorer le moral et les attitudes dans l'apprentissage du français, ainsi que l'orientation scolaire comprenant des diverses techniques efficaces pour apprendre le français. Cela devrait donc les aider à améliorer leurs compétences et à raccourcir leur période d'études.

La formulation du problème dans cette étude est : (1) Quel est l'intuition linguistique des étudiants "faibles" au département de pédagogie de français ? et (2) Quelles sont les stratégies d'apprentissage utilisées par les étudiants "faibles" au département de pédagogie de français ? Cette étude vise à décrire les intuitions linguistiques et les stratégies d'apprentissage utilisées par les étudiants 'faibles" au département de pédagogie de français.

Le talent est un potentiel de quelqu'un d'inné dès la naissance et non quelque chose de vraiment réel. Il est comme une possibilité à réaliser. D'après Suryabrata $(2009$, p. 174) le talent est une condition chez une personne qui lui permet, avec un exercice spécial, d'acquérir une compétence, des connaissances et des compétences spécifiques.

Une recherche sur le talent a été menée par Fitri (2014) sur le système d'information du traitement des données pour les résultats des tests d'orientation professionnelle dans les branches des sports de gymnastique.

Il y a des divers talents et chaque enfant en a, l'un d'entre eux est l'intuition linguistique. Il existe deux types d'intuitions linguistiques, ce sont l'intuition linguistique lié à l'acquisition de la langue maternelle (language acquisition) et l'intuition linguistique lié à l'apprentissage d'une langue seconde ou d'une langue étrangère (language learning) (Brown, 1989, p. 31 ; Vans, 1990, p. 177). Le talent de première langue est aussi appelé talent général car il appartient à chaque enfant. Chomsky l'appelle en abréviation LAD (Language Acquisition Device). Tous les enfants normaux naissent avec la capacité d'apprendre la langue.

Pour découvrir le talent de quelqu'un, on utilise un test de talent. C'est un test utilisé pour mesurer les capacités potentielles en faisant certaines activités, puis le succès de cette activité peut être prédit. Il y a quelques types de tests de talents, par exemples Flanagan Aptitudes Classification Test (FACT), General Aptitudes Test Battery (GATB), The Differential Aptitudes Test (DAT).

Flanagan Aptitudes Classification Test (FACT) a été créé par J.C. Flanagan. C'est une tentative d'obtenir un système de classification standard pour déterminer les talents et les capacités de base individuels sur certaines tâches. Cet outil se compose de 14 tests pouvant être utilisés en totalité ou séparément.

General Aptitudes Test Battery (GATB), composé par Odell dans les années 1960, se compose de 12 sous tests pouvant mesurer 9 capacités ou talents de base, ce sont : intelligence, aptitude verbale, nombre d'aptitude, espace d'aptitude, perception de la forme, perception cléricale, coordination motrice, dextérité des doigts et dextérité de la main.

The Differential Aptitudes Test (DAT) composé par Bennett, Seashore et Wesman en 1947.L'objectif de ce test est d'obtenir une procédure d'évaluation scientifique, intégrée et standardisée car le test d'IQ est insuffisante. Pour cette raison, il est nécessaire de créer un test pour mesurer des divers facteurs. Ce test comprend 8 types, ce sont: raisonnement verbal, raisonnement numérique, raisonnement abstrait, rapidité et précision de la perception, raisonnement mécanique, relations spatiales et utilisation du langage.

Dans cette étude, The Differential Aptitudes Test (DAT), a été utilisé, en particulier le test de capacité de réflexion verbale et le test de capacité de réflexion abstraite. Le test de capacité de réflexion verbale est conçu pour mesurer la capacité d'une personne à comprendre des idées et des concepts et à résoudre des problèmes exprimés sous forme de mots. La capacité de réflexion verbale est un facteur prédictif favorable à la performance individuelle dans l'apprentissage des langues et dans le domaine de la langue, comme les écrivains, les linguistes, et les orateurs. Les tests de capacité de réflexion abstraite sont conçus pour mesurer la capacité d'un individu à identifier rapidement les modèles, les règles logiques et les tendances des informations et à les utiliser pour résoudre des problèmes.

Les recherches sur les stratégies d'apprentissage des langues étrangères ont été menées par Sugeng (2004) dont le titre est "Stratégie d'apprentissage de l'anglais comme langue étrangère liée aux facteurs démographiques chez les apprenants adultes, Isyam (2011) dont le titre est "Stratégies d'apprentissage des langues étrangères", et Irfadila (2016) dont le titre est "Relation entre les stratégies de lecture et la capacité de comprendre le texte de lecture indonésien chez les étudiants des programmes d'études de langue et de littérature indonésiennes". 
Nunan (1991, p. 52) soutient que les stratégies d'apprentissage des langues étrangères sont des processus mentaux. Isyam (2011, pp. 87-93) résume qu'il existe quatorze stratégies d'apprentissage des langues étrangères:(1) trouver des stratégies d'apprentissage appropriées, (2) gérer des informations linguistiques et des programmes d'apprentissage, (3) avoir un pouvoir créatif, (4) rechercher des opportunités, (5) apprendre à vivre dans l'incertitude,(6) utiliser des choses qui aident la mémoire , (7) utiliser des erreurs, (8) utiliser des connaissances linguistiques, (9) permettre le contexte à aider (10) apprendre à deviner correctement, (11) étudier des phrases ou des expression dans l'ensemble, (12) apprendre les habitudes qui sont inhérent aux locuteurs natifs, (13) apprendre des techniques de parler, et (14) utiliser des styles de langue différents.

Oxford (1990, pp.16-21) divise d'abord les stratégies d'apprentissage en deux grandes parties :les stratégies directes et indirectes. Les stratégies directes sont ensuite réparties en trois types : la mémoire, le cognitif et la compensation. Les stratégies indirectes sont divisées en trois : métacognitive, affective et sociale. Ainsi, individuellement, il existe six types de stratégies, ce sont: mémoire, cognitive, compensation, métacognitive, affective et sociale ; et chaque stratégie a ses propres types d'activités.

Les stratégies d'apprentissage de la mémoire sont utilisées par les apprenants en utilisant des connaissances et des expériences d'apprentissage antérieures. Cette stratégie d'apprentissage implique beaucoup de mémoire et de processus d'apprentissage qui utilisent la mémoire. Cette stratégie comprend la répétition de la leçon précédente.

La stratégie cognitive est l'ensemble du comportement de l'élève dans le processus d'enseignement et d'apprentissage lié à l'utilisation du pouvoir de réflexion de l'apprenant. Cette stratégie peut prendre la forme de diverses activités. Le comportement cognitif qui décrit cette stratégie est la suivante: corriger ses propres erreurs, utiliser des gestes, s'exercer à prononcer des mots, écrire dans un cahier, lire d'un tableau et regarder des supports pédagogiques.

Les stratégies d'apprentissage de la compensation sont utilisées par les apprenants qui ont des compétences assez élevées. Cette stratégie d'apprentissage est généralement utilisée pour surmonter certaines limitations linguistiques. Ceux qui sont inclus dans ce type de stratégie d'apprentissage sont: déterminer ou choisir le sujet à discuter, essayer d'éviter les sujets difficiles.

Les stratégies métacognitives sont toutes des comportements d'apprenants liés aux tactiques ou aux moyens des apprenants pour affronter et gérer les matériels d'enseignement et d'apprentissage. Cette stratégie peut être tangible : concentrer l'attention, planifier et organiser les activités d'enseignement et d'apprentissage et évaluer le processus d'enseignement et d'apprentissage.

La stratégie affective désigne tout comportement de l'élève lié aux attitudes et aux sentiments des apprenants face au processus d'apprentissage. Cette stratégie est divisée en deux: affectif positif et affectif négatif. Les stratégies affectives positives sont représentées par quatre comportements: le rire qui montre du plaisir ou de la satisfaction, le sourire, la satisfaction et montrer du plaisir à cause de choses amusantes. Les stratégies affectives négatives sont représentées par cinq comportements : montrer de la confusion, se plaindre, ne pas donner l'attention à l'enseignant, parler à un ami du sujet autrement de la pertinence de l'apprentissage et montrer l'ignorance.

Les stratégies sociales sont tous des comportements des élèves liés à la coopération des apprenants avec leurs collègues pour atteindre les objectifs d'apprentissage. Cette stratégie se manifeste par six activités: parler avec des amis sur le sujet, aider des amis conformément aux activités d'enseignement et d'apprentissage, demander de l'aide aux amis, complimenter des amis, harceler ou encourager des amis et des amis dérangeants.

\section{Méthode}

Cette recherche a été menée au département de pédagogie de français, de la faculté des langues et des arts, Universitas Negeri Semarang. Les variables étudiées étaient les intuitions linguistiques et les stratégies d'apprentissage des étudiants du département de pédagogie de français qui sont "faibles".

Le modèle de recherche utilisé dans cette étude est celui de descriptif pour décrire les intuitions linguistiques et les stratégies d'apprentissage utilisées par les étudiants du département de pédagogie de français qui sont "faibles".

Dans cette étude, l'identification a été menée pour déterminer les étudiants qui sont "faibles", puis ils deviennent les répondants à la recherche à qui on a demandé de subir un test d'intuition linguistique et de remplir des instruments de recherche sur les stratégies d'apprentissage. D'après les résultats de l'identification, il a été obtenu 10 étudiants du second semestre et 10 étudiants du quatrième semestre. Le nombre de répondants dans cette étude était donc de 20. 
Les données de cette étude ont été recueillies à l'aide de tests d'intuition linguistique et de questionnaires sur les stratégies d'apprentissage utilisées par les étudiants pour étudier le français. Les tests d'intuitions linguistiques sont organisés dans le laboratoire d'orientation et de conseil, Faculté d'Education, Universitas Negeri Semarang par des enseignants ayant un certificat d'examinateur, utilisant les instruments de test standardisés. Les capacités testées incluent la capacité de réflexion verbale et la capacité de réflexion abstraite. Le questionnaire sur les stratégies d'apprentissage est construit de la théorie d'Oxford (1990, pp. 16-21) sur les stratégies d'apprentissage des langues étrangères qui incluent des stratégies directes et indirectes. La grille du questionnaire est présentée cidessous.

Tableu 3 La grille du questionnaire

\begin{tabular}{|c|c|c|c|}
\hline $\begin{array}{l}\text { Nom de la } \\
\text { stratégie }\end{array}$ & Indicateur & Sous indicateur & $\begin{array}{l}\text { Numéro } \\
\text { d'article }\end{array}$ \\
\hline \multicolumn{4}{|c|}{ Stratégie directe } \\
\hline \multirow{7}{*}{$\begin{array}{l}\text { Stratégie de la } \\
\text { mémoire }\end{array}$} & \multirow{3}{*}{$\begin{array}{l}\text { Créer des connexions } \\
\text { mentales }\end{array}$} & Grouper/ classifier & 1,2 \\
\hline & & Lier / décrire & 3,4 \\
\hline & & Appliquer au contexte & 5 \\
\hline & \multirow{3}{*}{ Utiliser des images et des sons } & utiliser des images & 6 \\
\hline & & Utiliser des mots-clés & 7 \\
\hline & & $\begin{array}{l}\text { Imagerie des sons du langage en } \\
\text { mémoire }\end{array}$ & 8 \\
\hline & Utiliser l'action & $\begin{array}{l}\text { Répéter un acte physique ou } \\
\text { l'associer à une sensation physique }\end{array}$ & 9,10 \\
\hline \multirow{12}{*}{$\begin{array}{l}\text { Stratégie } \\
\text { cognitive }\end{array}$} & \multirow[t]{3}{*}{ Pratiquer la langue apprise } & $\begin{array}{l}\text { Capable de reconnaître et d'utiliser } \\
\text { des formules et des modèles }\end{array}$ & 11,12 \\
\hline & & capable de combiner & 13 \\
\hline & & $\begin{array}{llll}\begin{array}{l}\text { Pratiquer le langage } \\
\text { situations authentiques }\end{array} & & \text { des } \\
\end{array}$ & 14 \\
\hline & \multirow[b]{2}{*}{$\begin{array}{l}\text { Capable de recevoir et de } \\
\text { livrer des messages }\end{array}$} & Comprendre rapidement & 15 \\
\hline & & $\begin{array}{l}\text { Utiliser des ressources pour livrer et } \\
\text { recevoir des messages }\end{array}$ & 16,17 \\
\hline & \multirow{4}{*}{$\begin{array}{l}\text { Capable d'analyser et de } \\
\text { raisonner }\end{array}$} & $\begin{array}{l}\text { Capable de raisonner et de tirer des } \\
\text { conclusions }\end{array}$ & 18,19 \\
\hline & & Comparer avec les langues connues & 20 \\
\hline & & Capable de traduire des messages & 21 \\
\hline & & Capable de transférer des messages & 22 \\
\hline & \multirow{3}{*}{ Capable de structurer } & Toujours prendre des notes & 23 \\
\hline & & Capable de faire un résumé & 24 \\
\hline & & $\begin{array}{l}\text { Capable de reconnaître les parties } \\
\text { importantes (souligner) }\end{array}$ & 25 \\
\hline \multirow{8}{*}{$\begin{array}{l}\text { Stratégie de la } \\
\text { compensation }\end{array}$} & $\begin{array}{l}\text { Capable de deviner le sens } \\
\text { intelligemment }\end{array}$ & Capable d'utiliser l'index linguistique & 26 \\
\hline & \multirow{7}{*}{$\begin{array}{l}\text { Capable de surmonter les } \\
\text { difficultés à la fois orales et } \\
\text { écrites }\end{array}$} & Utiliser la langue maternelle & 27 \\
\hline & & $\begin{array}{l}\text { Ne pas hésiter à demander l'aide aux } \\
\text { autres }\end{array}$ & 28 \\
\hline & & $\begin{array}{l}\text { Utiliser l'expression ou la langue des } \\
\text { signes }\end{array}$ & 29,30 \\
\hline & & $\begin{array}{l}\text { Eviter la communication partielle ou } \\
\text { totale }\end{array}$ & 31,32 \\
\hline & & $\begin{array}{l}\text { Capable d'ajuster ou de modifier les } \\
\text { messages }\end{array}$ & 33 \\
\hline & & Capable d'estimer des mots & 34 \\
\hline & & Capable de paraphraser & 35 \\
\hline \multicolumn{4}{|c|}{ Stratégie indirecte } \\
\hline & Focus sur l'apprentissage & $\begin{array}{l}\text { Tester le matériel à étudier / } \\
\text { connecté avec ce qui est connu }\end{array}$ & $36,37,38$ \\
\hline
\end{tabular}




\begin{tabular}{|c|c|c|c|}
\hline \multirow{8}{*}{$\begin{array}{l}\text { Stratégie } \\
\text { métacognitive }\end{array}$} & & Faire attention & 39 \\
\hline & \multirow{5}{*}{$\begin{array}{l}\text { Planifier et organiser } \\
\text { l'apprentissage }\end{array}$} & $\begin{array}{l}\text { Découvrez comment apprendre les } \\
\text { langues }\end{array}$ & 40 \\
\hline & & Être organisé & 41 \\
\hline & & Atteindre l'objectif sincèrement & 42 \\
\hline & & $\begin{array}{ll}\begin{array}{l}\text { Planifier } \\
\text { d'apprentissage }\end{array} & \text { processus } \\
\end{array}$ & 43 \\
\hline & & $\begin{array}{l}\text { Chercher des occasions de pratiquer } \\
\text { la langue apprise }\end{array}$ & 44 \\
\hline & \multirow[t]{2}{*}{ Évaluer comment apprendre } & Se surveiller & 45 \\
\hline & & S'évaluer & 46 \\
\hline \multirow{6}{*}{$\begin{array}{l}\text { Stratégie } \\
\text { affective }\end{array}$} & \multirow{3}{*}{$\begin{array}{l}\text { Capable de surmonter } \\
\text { l'anxiété }\end{array}$} & $\begin{array}{l}\text { Faire la relaxation, respirer } \\
\text { profondément ou faire la méditation }\end{array}$ & $47,48,49$ \\
\hline & & Écouter de la musique & 50 \\
\hline & & Avoir le sens de l'humour & 51 \\
\hline & \multirow{2}{*}{$\begin{array}{l}\text { Capable de surmonter } \\
\text { l'anxiété }\end{array}$} & $\begin{array}{l}\begin{array}{l}\text { S'apprécier pour les } \\
\text { accomplis }\end{array} \\
\end{array}$ & 52 \\
\hline & & Se féliciter & 53 \\
\hline & Utiliser le goût & Faire un journal quotidien & 54 \\
\hline \multirow{5}{*}{ Stratégie sociale } & \multirow[b]{2}{*}{ Poser des questions } & Assurer et clarifier la vérité & 55 \\
\hline & & Vouloir accepter la correction & 56 \\
\hline & \multirow[b]{2}{*}{$\begin{array}{l}\text { Vouloir travailler avec les } \\
\text { autres }\end{array}$} & Capable de travailler avec ses amis & 57 \\
\hline & & $\begin{array}{l}\text { Capable de travailler avec des } \\
\text { locuteurs natifs }\end{array}$ & 58 \\
\hline & $\begin{array}{l}\text { Cultiver l'empathie pour les } \\
\text { autres }\end{array}$ & Ouvert à la culture des autres & 59 \\
\hline
\end{tabular}

Les données collectées se présentent sous la forme de résultats aux tests d'intuitions linguistiques et aux résultats des questionnaires de la stratégie d'apprentissage en français des répondants décrits en utilisant les catégories suivantes :

1. Capacité de réflexion verbale et de réflexion abstraite

Tableu 4 Classification de niveau de capacité de réflexion verbale et de réflexion abstraite

\begin{tabular}{|l|l|}
\hline Score & Catégorie \\
\hline $75-100$ & Élevée \\
\hline $50-74$ & Moyenne \\
\hline $0-49$ & Base \\
\hline
\end{tabular}

\section{Stratégie d'apprentissage}

Les données sur les stratégies d'apprentissage ont été analysées à l'aide d'une analyse descriptive en pourcentage.

\section{Résultat et discussion}

Au moment de la collecte des données, 2 répondants étaient absents, de sorte que les données n'avaient été obtenues que de 18 répondants. Les données collectées sont analysées en utilisant le critère du tableau 2 et l'analyse descriptive en pourcentage. Voici les résultats.

1. Test d'intuition linguistique

Les compétences testées au test d'intuition linguistique dans cette étude étaient la capacité de réflexion verbale et la capacité de réflexion abstraite. Les données obtenues sur ce test sont les suivantes. 
Tableu 5 Score de résultats du test d'intuition linguistique

\begin{tabular}{|l|l|l|}
\hline No Répondant & Score CRV & Score CRA \\
\hline 1 & 45 & 10 \\
\hline 2 & 85 & 60 \\
\hline 3 & 5 & 3 \\
\hline 4 & 85 & 30 \\
\hline 5 & 40 & 20 \\
\hline 6 & 65 & 30 \\
\hline 7 & 45 & 60 \\
\hline 8 & 80 & 70 \\
\hline 9 & 15 & 20 \\
\hline 10 & 55 & 70 \\
\hline 11 & 20 & 15 \\
\hline 12 & 75 & 85 \\
\hline 13 & 10 & 15 \\
\hline 14 & 1 & 20 \\
\hline 15 & 85 & 65 \\
\hline 16 & 25 & 55 \\
\hline 17 & 85 & 75 \\
\hline 18 & 60 & 65 \\
\hline
\end{tabular}

Explication :

CRV : Capacité de Réflexion Verbale

CRA : Capacité de Réflexion Abstraite

Les données du Table 5 sont ensuite analysées en utilisant le critère du tableau 2 afin de produire des informations dans Table 6 et 7 .

Tableu 6 Résultats du test de capacité de réflexion verbal

\begin{tabular}{|c|c|c|c|}
\hline Score & Fréquence & Pourcentage & Catégorie \\
\hline $75-100$ & 6 & $33,3 \%$ & Élevée \\
\hline $50-74$ & 3 & $16,7 \%$ & Moyenne \\
\hline $0-49$ & 9 & $50 \%$ & Base \\
\hline
\end{tabular}

Basé sur ce résultat, on peut faire le diagramme suivant.

\section{Distribution du résultat du test de capacité de réflexion verbale}

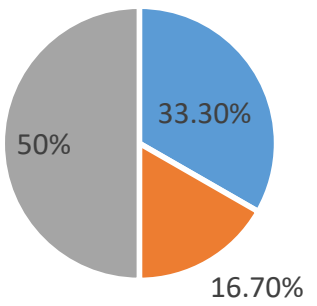

- Élevée - Moyenne " Base

Figure 1 Distribution du résultat du test de capacité de réflexion verbale 
Basé sur les données du Table 6etsur le Fig. 1, on peut constater que $50 \%$ des répondants appartiennent à la catégorie de Base, 16,7\% dans la catégorie de Moyenne et 33,3\% dans la catégorie d'Élevée. On peut donc dire que les résultats de la capacité de réflexion verbale sont dominés par les répondants appartenant à la catégorie de Base.

Le Résultat du test de capacité de réflexion abstraite vu dans le Table 7.

Tableu 7 Résultats du test de capacité de réflexion abstraite

\begin{tabular}{|c|c|c|c|}
\hline Score & Fréquence & Pourcentage & Catégorie \\
\hline $75-100$ & 2 & $11,1 \%$ & Élevée \\
\hline $50-74$ & 7 & $38,9 \%$ & Moyenne \\
\hline $0-49$ & 9 & $50 \%$ & Base \\
\hline
\end{tabular}

Basé sur ce résultat, on peut faire le diagramme suivant.

\section{Distribution du résultat du test de capacité de réflexion abstraite}

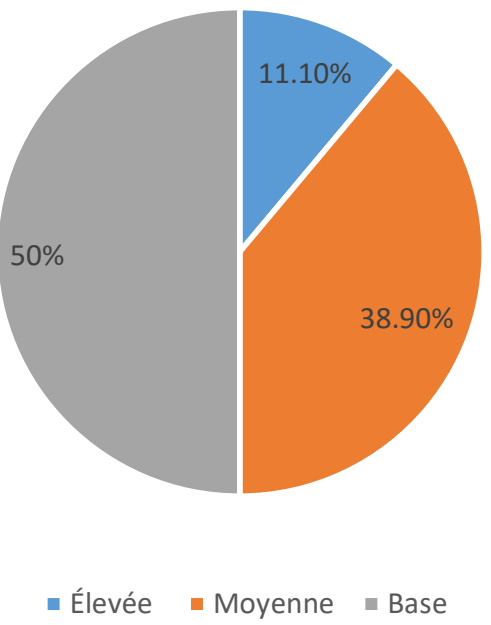

Figure 2 Distribution du résultat du test de capacité de réflexion abstraite

Basé sur les données du Table 7 et sur le Fig. 2, on peut constater que $50 \%$ des répondants appartiennent à la catégorie de Base, 38,9\% dans la catégorie de Moyenne et 11,1\% dans la catégorie d'Élevée. On peut donc dire que les résultats de la capacité de réflexion abstraite sont dominés par les répondants appartenant à la catégorie de Base.

2. Questionnaire sur la stratégie d'apprentissage

Les données sur les stratégies d'apprentissage utilisées par les répondants pour apprendre le français ont été obtenues à partir de questionnaires construits à partir de la théorie d'Oxford sur les stratégies d'apprentissage des langues étrangères qui comprend des stratégies directes et indirectes et leurs descriptions, ce sont : stratégies de la mémoire, stratégies cognitives, stratégies de la compensation, stratégies métacognitives, stratégies affectives, et stratégie sociale. Le questionnaire comprend 59 éléments de déclarations. Les résultats de l'analyse des données du questionnaire sont présentés dans le Tableu 8.

Tableu 8 Résultats du questionnaire sur les stratégies d'apprentissage

\begin{tabular}{|l|l|l|}
\hline Stratégies & Score & Score Total \\
\hline Stratégie Directes & \\
\hline
\end{tabular}




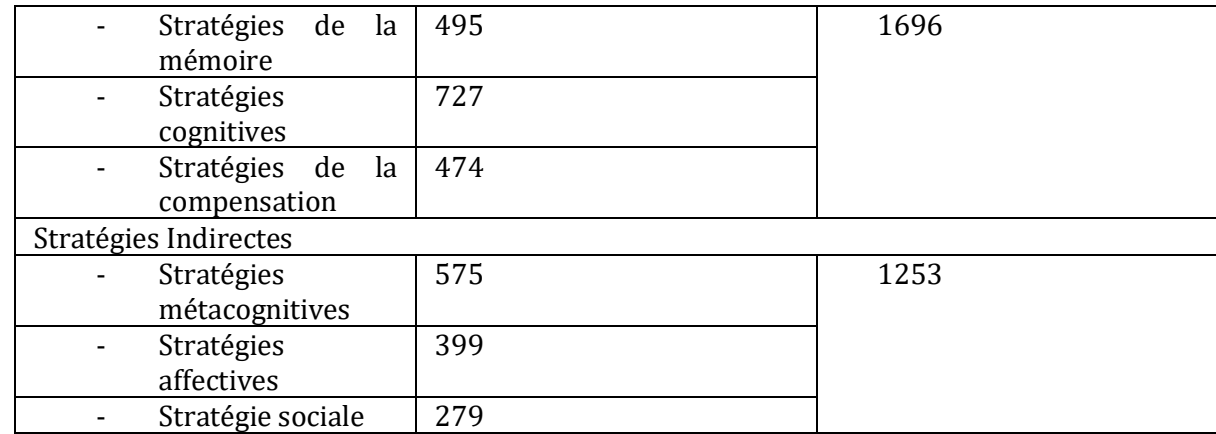

D'après le Table 8 , on peut dire que les répondants utilisent toutes les stratégies d'apprentissage des langues. Les données du Table 6 ont été analysées en utilisant l'analyse descriptive en pourcentage pour produire des informations dans les Fig. 3 et 4.

\section{Utilisation de stratégies directes et indirectes}

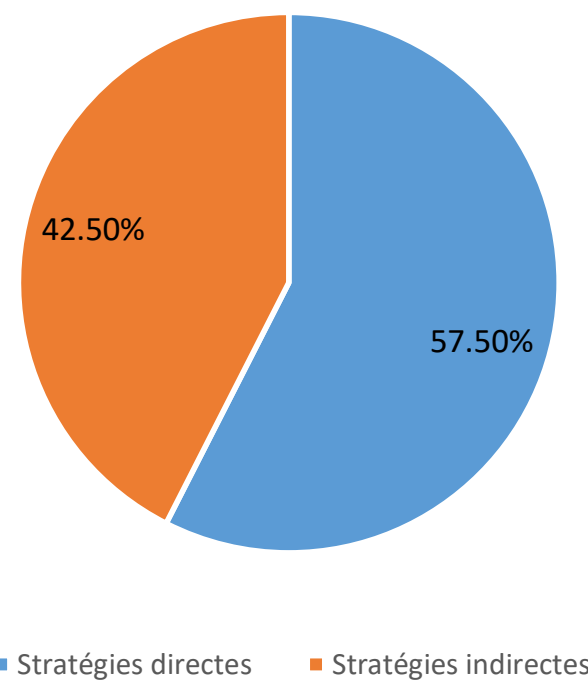

Figure 3 Utilisation de stratégies directes et indirectes

Dans le Fig. 3, on peut voir que la stratégie directe est utilisée par 57,7\% des répondants, tandis que la stratégie indirecte est utilisée par $42,5 \%$ des répondants. L'utilisation des stratégies d'apprentissage des langues plus détaillée est présentée dans le Fig. 4. 


\section{Distribution d'utilisation de la stratégie d'apprantissage}

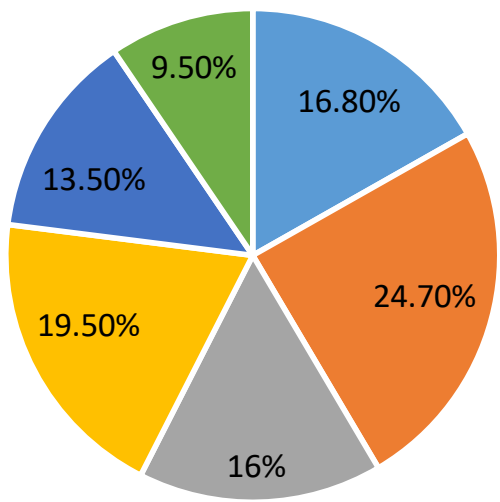

- stratégies de la mémoire

v stratégies métacognitives

$$
\begin{aligned}
& \text { - stratégies cognitives } \\
& \text { - stratégies affectives }
\end{aligned}
$$

- stratégies de la compensation

- stratégie sociale

Figure 4 Distribution d'utilisation de la stratégie d'apprentissage

Basé sur le Fig. 4, on peut dire que 24,70 \% des répondants utilisent la stratégie cognitive, 19,50 \% la stratégie métacognitive, 16,80 \% stratégie de la mémoire, 16 \% stratégie de la compensation, $13,50 \%$ la stratégie affective, et 9,50 \% stratégie sociale. On peut donc affirmer que la stratégie la plus utilisée par les répondants est celle de cognitive et que la moins utilisée est celle de sociale.

\section{Conclusion}

Basé sur les résultats de l'analyse des données, on peut conclure que :

1. Les intuitions linguistiques des étudiants "faibles" au département de pédagogie de français qui sont compensées par des tests de capacité de réflexion verbale et de réflexion abstraite, montrent que $50 \%$ se situent dans la catégorie de Base.

2. La stratégie d'apprentissage des langues étrangères utilisée par les étudiants "faibles" au département de pédagogie de français comprend : stratégie de la mémoire, cognitive, de la compensation, métacognitive, affective, et sociale. La stratégie la plus utilisée par les répondants est celle de cognitive et la moins utilisée est celle de sociale.

\section{Références}

Brown, A. L., \& Palinscar, A. S. (1989). Guided, cooperative learning and individual knowledge acquisition.

In L. B. Resnick (Ed.), Dans Knowing, Learning and Instruction Essays in Honour of Robert Glaser (pp. 393-452). Hillsdale: Psychology Press.

Fitri, M. (2010). Sistem Informasi Pengolahan Data Hasil Tes Pemanduan Bakat Cabang Olahraga Senam.

Jurnal Penelitian Pendidikan, 14(2), 16-27. https://doi.org/10.17509/manajerial.v9i1.1197

Irfadila, M. S. (2016). Hubungan Strategi Membaca dengan Kemampuan Memahami Teks Bahasa

Indonesia Mahasiswa Prodi Pendidikan Bahasa dan Sastra Indonesia FKIP UMSB Padangpanjang

Jurnal Gramatika, 1(1), 57-71. https://doi.org/https://doi.org/10.22202/jg.2015.v1i1.1157

Isyam, A. (2011). Strategi-Strategi Belajar Bahasa Asing. Lingua Didaktika, 4(2), 86-95.

https://doi.org/https://doi.org/10.24036/ld.v4i2.1259 
Nunan, D. (1991). Language Teaching Methodology: A textbook for teachers. Hertfordshire: Prentice Hall.

Oxford, R. L. (1990). Language Learning Strategies: What Every Teacher Should Know. New York: Newbury House Publisher.

Sugeng, B. (2004). Strategi Belajar Bahasa Inggris sebagai Bahasa Asing Kaitannya dengan Faktor-Faktor Demografik pada Pembelajar Dewasa. Diksi, 11(1). Retrieved from http://eprints.uny.ac.id/4810/1/Strategi_BelajarBahasa_Inggris.pdf Suryabrata, S. (2009). Psikologi Pendidikan. Jakarta: Grafindo Persada.

Vann, R. J., \& Abraham, R. G. (1990). Strategies of Unsuccessful Language Learners. TESOL Quarterly, 24(2), 177-198. https://doi.org/10.2307/3586898 